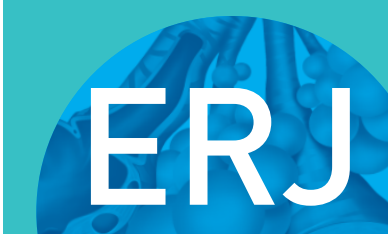

open research

\section{Prospective multicentre study on the safety and utility of transbronchial lung cryobiopsy with endobronchial balloon}

\author{
Minoru Inomata (10 ${ }^{1}$, Naoyuki Kuse ${ }^{1}$, Nobuyasu Awano ${ }^{1}$, Mari Tone ${ }^{1}$, \\ Hanako Yoshimura ${ }^{1}$, Tatsunori Jo', Jonsu Minami ${ }^{1}$, Kohei Takada', Bae Yuan², \\ Toshio Kumasaka ${ }^{2}$, Hideaki Yamakawa ${ }^{3}$, Shintaro Sato ${ }^{3}$, Kazunori Tobino ${ }^{4}$, \\ Hidekazu Matsushima ${ }^{3}$, Tamiko Takemura ${ }^{2,5}$ and Takehiro Izumo ${ }^{1}$
}

Affiliations: ${ }^{1}$ Dept of Respiratory Medicine, Japanese Red Cross Medical Center, Shibuya, Japan. ${ }^{2}$ Dept of Pathology, Japanese Red Cross Medical Center, Shibuya, Japan. ${ }^{3}$ Dept of Respiratory Medicine, Saitama Red Cross Hospital, Saitama, Japan. ${ }^{4}$ Dept of Respiratory Medicine, lizuka Hospital, lizuka, Japan. ${ }^{5}$ Dept of Pathology, Kanagawa Cardiovascular and Respiratory Center, Yokohama, Japan.

Correspondence: Minoru Inomata, Dept of Respiratory Medicine, Japanese Red Cross Medical Center, 4-1-22 Hiroo Shibuya-ku, Tokyo 150-8935, Japan. E-mail: inomataminoruanms.ac.jp

ABSTRACT Transbronchial lung cryobiopsy (TBLC) has been increasingly utilised to diagnose diffuse parenchymal lung diseases (DPLDs) and lung cancers; however, TBLC protocols have not yet been standardised and the rate of complications associated with this procedure vary widely. Therefore, this prospective multicentre observational study investigated the safety and utility of the TBLC technique in patients with diffuse and localised respiratory diseases.

This study was conducted at multiple medical centres in Japan between July 2018 and April 2019. The study's primary end-point was the rate of severe or serious adverse events associated with TBLC. Adverse events included bronchial bleeding, pneumothorax, pneumonia, respiratory failure, and an acute exacerbation of interstitial pneumonia. Adverse events were graded according to severity. During the TBLC procedure, an endobronchial balloon catheter for bronchial blockade was used in all patients. Pathological confidence and quality of specimens were categorised into three groups.

A total of 112 patients were included. Neither severe nor serious adverse events were identified; therefore, the primary end-point was met. Nineteen patients (17\%) experienced no bronchial bleeding. Mild or moderate bronchial bleeding was identified in $67 \%$ and $16 \%$ of patients, respectively. Mild pneumothoraces were identified in four patients (3.6\%). The safety profile in patients aged $\geqslant 75$ years was not significantly different from younger patients. Definite or probable pathological diagnoses were made in $84.9 \%$ of patients.

This TBLC protocol with routine use of an endobronchial balloon had an acceptable safety profile and diagnostic yield in patients, including elderly ones, with diffuse and localised respiratory diseases.

@ERSpublications

This multicentre prospective study showed an acceptable safety profile and diagnostic yield for transbronchial cryobiopsy with routine use of an endobronchial balloon for patients with diffuse and localised respiratory diseases, including elderly patients https://bit.ly/2Vv8Gky

Cite this article as: Inomata $\mathrm{M}$, Kuse $\mathrm{N}$, Awano $\mathrm{N}$, et al. Prospective multicentre study on the safety and utility of transbronchial lung cryobiopsy with endobronchial balloon. ERJ Open Res 2020; 6: 00008-2020 [https://doi.org/10.1183/23120541.00008-2020].

Received: 7 Jan 2020 | Accepted after revision: 7 April 2020

Copyright $\odot$ ERS 2020. This article is open access and distributed under the terms of the Creative Commons Attribution Non-Commercial Licence 4.0. 


\section{Introduction}

Transbronchial lung cryobiopsy (TBLC) is a novel technique for the collection of lung tissue biopsies of high quality and large size [1,2], with reduced artefacts [1,3] and improved diagnostic yields in comparison to forceps biopsies of lung tissue $[1,4]$.

TBLC has been increasingly utilised to diagnose diffuse parenchymal lung diseases (DPLDs) [2, 5]. TBLC increases diagnostic confidence in the multidisciplinary diagnoses of interstitial lung diseases and may prove useful for the diagnosis of idiopathic pulmonary fibrosis (IPF) [6]. TBLC has also been successfully used for diagnoses of peripheral localised pulmonary lesions, especially lung tumours [7].

Efficacy, safety, and feasibility of TBLC in daily clinical practice has been reported in patients with various lung diseases [8]. However, TBLC procedure protocols are yet to be standardised, resulting in significant variations in diagnostic yields and complication rates at multiple centres.

One of the major identified complications of TBLC is bleeding. Although the rate of clinically relevant bleeding is higher with TBLC than with forceps biopsies $[9,10]$, use of an endobronchial balloon reduces the frequency of this complication [11]; therefore, bronchial blockade is a key component of maintaining haemostasis during this procedure. This multicentre prospective study investigated the procedural outcomes and utility of using TBLC with endobronchial balloon placement in adults with diffuse or localised respiratory diseases.

\section{Materials and methods}

Ethical considerations

This prospective multicentre study design and protocol was approved by the Institutional Review Board of the Japanese Red Cross Medical Center (No. 901) and confirmed by the Ethics Committee of each individual site. It was registered on the University Hospital Medical Information Network (UMIN 000033284). Written informed consent was obtained from all patients.

\section{Study design and population}

This study was conducted at the Department of Respiratory Medicine at the Japanese Red Cross Medical Center, Saitama Red Cross Hospital, and Iizuka Hospital between July 2018 and August 2019 in Japan. Patient inclusion criteria were as follows: age $>20$ years, suspected or diagnosed DPLDs or malignant tumours in the lung periphery, and ability to give informed consent. Patient exclusion criteria were as follows: allergies to lidocaine, midazolam, flumazenil, or pethidine; high risk of bronchial bleeding due to suspected bronchial artery aneurysms or lung metastases of renal cell carcinoma; unsuitability for withholding of anticoagulation therapy for 1 to 7 days, depending on the type of anticoagulant drug; unstable severe comorbidities, including unstable angina, congestive heart failure, or severe bronchial asthma; and pregnancy. Patients were consecutively enrolled in the study if they were qualified based on these inclusion and exclusion criteria and if a pulmonologist at their hospital determined that TBLC was needed for diagnostic purposes, including patients with consultation for this procedure. Prior to TBLC, several variables were evaluated, including the smoking index, usage of antithrombotic drugs, results of pulmonary function testing, and levels of bleeding parameters, fibrotic markers, and tumour markers. These variables were used to determine the baseline respiratory status and risk of adverse events of patients, as well as to support their initial diagnosis.

\section{Procedural protocol}

Bronchoscopy was performed with a flexible bronchoscope of an EB-580T, EB-580S (Fujifilm, Tokyo, Japan), BF-1T290, BF1T-260, or BF-260 (Olympus, Tokyo, Japan). Patients underwent intravenous deep anaesthesia with pethidine, midazolam, or fentanyl, and $2 \%$ lidocaine was administered intratracheally. A flexible endotracheal tube (SACETT suction above cuff endotracheal tube 8.0-8.5 mm; Smiths Medical International Ltd., Minneapolis, MN, USA) was inserted for airway control. An endobronchial balloon (Fogarty ${ }^{\oplus}$ catheter, E-080-4F; Edwards Life-sciences, Irvine, CA, USA) was used for bronchial blockade and for haemostasis in all patients. If necessary, forceps (FB-15C-1, FB-231D; Olympus, Tokyo, Japan) and a guide sheath (K-201, K-203; Olympus, Tokyo, Japan) were used. A 1.4-mm 20-MHz radial probe (PB2020-M; Fujifilm or UM-S20-17S; Olympus) was also used in some patients for visualisation of lesions and blood vessels during determination of biopsy sites. All anticoagulant drugs were discontinued prior to the procedure as per guidelines [12].

\section{Endobronchial balloon insertion}

A deflated endobronchial balloon was inserted into the suction channel on the cuff of the endotracheal tube. After intubation, under moderate to deep sedation, the mobility of the endobronchial balloon without resistance was confirmed, and the endotracheal tube was then fixed. Before performing TBLC, the 
endobronchial balloon was placed in the segmental or subsegmental bronchus chosen for TBLC for control of bleeding and was then fixed during the TBLC procedure.

\section{TBLC procedure}

The flexible cryoprobe, with either a 1.9-mm or 2.4-mm diameter (ERBECRYO 2 system; Erbe Elektromedizin $\mathrm{GmbH}$, Tubingen, Germany), was advanced into a peripheral airway of the bronchus chosen for TBLC. Using radiography guidance, the cryoprobe was advanced until the pleural surface was reached and then withdrawn 1-2 cm proximally in patients with diffuse respiratory lesions. The probe was also advanced until the localised lesion was reached in patients with suspected malignant tumours, and then frozen for 5-7 s. The probe with the attached lung specimen and the bronchoscope were then quickly removed simultaneously. After withdrawal of the probe and the bronchoscope, the endobronchial balloon was prophylactically inflated with $2-3 \mathrm{~mL}$ of air from a $5-\mathrm{mL}$ syringe attached to the proximal site of the balloon. The cryoprobe with the attached lung specimen was submerged in a saline-filled pot at room temperature. The specimen was rapidly thawed and released. The bronchoscope was then returned to the biopsy site. After $60 \mathrm{~s}$ of inflation, the balloon was deflated to identify ongoing bleeding and $2 \mathrm{~mL}$ of an epinephrine-saline mixture ( $1 \mathrm{~mL}$ of epinephrine with $19 \mathrm{~mL}$ of saline) was administered. More epinephrine-saline mixture and/or ice-cold saline were instilled at the biopsy site if necessary. During the above procedure, a second proceduralist inflated the specimen with a $20-\mathrm{mL}$ syringe containing a small amount of saline for 60-120 s for histopathological examination [13], and then transferred it to $10 \%$ formalin. The process was then repeated to obtain multiple TBLC specimens. This TBLC protocol was used for both diffuse and localised lesions.

\section{Displacement of endobronchial balloon}

If the inflated endobronchial balloon was displaced to another bronchus where it could not block bleeding, it was defined as a major displacement (e.g. from the right $\mathrm{B}^{3} \mathrm{~b}$ to the right main bronchus). If the inflated endobronchial balloon was displaced, but it could still maintain its haemostatic effect, it was defined as a minor displacement (e.g. from the right $\mathrm{B}^{3}$ a to the entrance of the superior lobar bronchus).

\section{End-points}

Primary end-point

The primary end-point of this study was the rate of severe and serious adverse events associated with TBLC.

\section{Adverse events}

We categorised adverse events in the present study as follows: 1) bronchial bleeding, 2) pneumothorax, 3) pneumonia, 4) respiratory failure, and 5) acute exacerbation of interstitial pneumonia.

\section{Grading of adverse events}

The Clavien-Dindo classification system has been used to evaluate the severity of surgical complications [14]. In the present study, the grade of each adverse event was defined according to its severity (mild, moderate, severe, or serious) based on a modified form of the Clavien-Dindo classification system. For all types of adverse events, "serious" adverse events were defined as life-threatening consequences, and "severe" adverse events were defined as those requiring surgical or radiological interventions or invasive positive-pressure ventilation. "Moderate" and "mild" adverse events of different types were defined as follows: bronchial bleeding: moderate (cold saline or epinephrine needed three times or more) and mild (other than those indicated above); pneumothorax: moderate (chest tube placement indicated) and mild (other than those indicated above); pneumonia, respiratory failure, or an acute exacerbation of interstitial pneumonia: moderate (noninvasive positive-pressure ventilation indicated) and mild (drug therapy indicated or oxygen therapy (nasal cannula, reservoir nasal cannula, mask, or reservoir mask) needed for $>24 \mathrm{~h}$ for any cause).

\section{Secondary end-points}

Secondary end-points included the rate of severe or serious adverse events in patients $\geqslant 75$ years of age, the rate of all adverse events, the frequency of a definite or a probable diagnosis, and any artefacts of TBLC specimens.

\section{Pathological evaluation and diagnosis}

An expert pathologist evaluated TBLC specimens for pathological quality, quantity, and confidence, according to previous reports $[8,15]$. The quality and quantity of the tissue specimens were classified into three grades. Grade A specimens were defined as having an adequate amount of lung tissues corresponding to lesions on high-resolution computed tomography imaging. Grade $\mathrm{C}$ was defined as 
specimens that were difficult to evaluate because of a small quantity or lack of lesions. Grade B specimens were between grades A and C. The pathological confidence was also classified into three levels. Definite pathological diagnoses could be made with level A specimens. Diagnoses were difficult with Level C specimens. Level B specimens fell between levels A and C, and probable diagnoses could be determined.

\section{Multidisciplinary discussion diagnosis}

The final diagnosis for each patient was achieved through a multidisciplinary discussion (MDD). The diagnostic yield of TBLCs for MDD diagnoses was evaluated in all patients and, more specifically, in patients with DPLD and with malignant tumours.

\section{Sample size}

A sample size of 98 was calculated using an expected value of 0.10 and a threshold of 0.20 with a two-tailed statistical significance of $\alpha=0.05$ and a power of at least 0.80 . Allowing for $10 \%$ missing data (due to patient dropout), we planned to recruit 110 patients to the present study.

\section{Statistical analyses}

Continuous variables are presented as medians and ranges, and categorical variables are presented as absolute numbers and percentages. The rate of adverse events and the frequency of definite or probable diagnoses were analysed in all patients. A chi-squared test or Fisher's exact test were used for categorical variables. Data were analysed using JMP 9 version 9.0.3 (SAS Institute Inc., Cary, NC, USA). A two-tailed p-value $<0.05$ was considered statistically significant.

\section{Results}

\section{Patient characteristics}

For this multicentre prospective study, 112 patients were recruited from the Japanese Red Cross Medical Center, Saitama Red Cross Hospital, and Iizuka Hospital. Baseline patient characteristics are shown in table 1. Eighty-seven patients had diagnosed DPLD, and 25 patients had suspected or diagnosed malignant lung tumours.

There were no significant differences in the number of patients who were being treated with antithrombotic drugs, the levels of thrombocytes, or the activated partial thromboplastin times between patients with DPLD and those with malignant tumours. The prothrombin time (INR), however, was

\section{TABLE 1 Baseline patient characteristics}

\begin{tabular}{|c|c|c|c|c|}
\hline & Total $(n=112)$ & DPLD (n=87) & Malignant tumour $(n=25)$ & p-value \\
\hline Sex female/male $n / n$ & $49 / 63$ & $37 / 50$ & $12 / 13$ & 0.627 \\
\hline Weight kg & $58(37.4-96.1)$ & $60.1(37.4-96.1)$ & $54.2(39.6-75)$ & 0.093 \\
\hline Smoking index pack-years & $31(0.3-160)$ & $30(0.3-120)$ & $40(5-65)$ & 0.033 \\
\hline Antithrombotic drug & $12(10.7)$ & $10(11.1)$ & $2(9.1)$ & 0.607 \\
\hline Activated partial thromboplastin time s & $30.9(21.4-66.6)$ & $31.9(21.4-66.6)$ & $28.1(22.6-58.5)$ & 0.079 \\
\hline $\mathrm{KL}-6 \mathrm{U} \cdot \mathrm{mL}^{-1}$ & $560(104-9516)$ & $584(104-9516)$ & - & - \\
\hline SP-D ng.mL ${ }^{-1}$ & $175.5(17.2-1050)$ & $173(7.6-1050)$ & - & - \\
\hline CEA $\mathrm{ng} \cdot \mathrm{mL}^{-1}$ & $4.4(1-507)$ & - & $4.4(1-507)$ & - \\
\hline CYFRA $\mathrm{ng} \cdot \mathrm{mL}^{-1}$ & $1.8(0.6-71.9)$ & - & $1.8(0.6-71.9)$ & - \\
\hline ProGRP pg.mL $\mathrm{mL}^{-1}$ & $44.5(23.1-206)$ & - & $44.5(23.1-206)$ & - \\
\hline$D_{\text {Lco }} \%$ pred & $79.9(14.9-197.4)$ & $73.1(16.4-155.8)$ & $99.85(14.86-197.4)$ & 0.033 \\
\hline
\end{tabular}

Data are presented as $\mathrm{n}(\%)$ or median (range), unless otherwise stated. DPLD: diffuse parenchymal lung disease; INR: international normalised ratio; KL-6: Krebs von den Lungen-6; SP-D: surfactant protein-D; CEA: carcinoembryonic antigen; CYFRA: cytokeratin 19 fragment; ProGRP: progastrin releasing peptide; FVC: forced vital capacity; $F E V_{1}$ : forced expiratory volume in $1 \mathrm{~s} ; D_{\text {Lco: }}$ diffusing capacity for carbon monoxide; \% pred: \% predicted. 
significantly higher in patients with DPLD, potentially as a complication of their antithrombotic drug usage. These patients did not, however, have an increased bleeding tendency.

The smoking index was significantly lower in patients with DPLD than in patients with malignant lung tumours; however, there was no significant difference between the forced expiratory volume in $1 \mathrm{~s}\left(\mathrm{FEV}_{1}\right)$ of these two patient groups. The forced vital capacity (FVC), \% predicted FVC, and \% predicted diffusing capacity of the lungs for carbon monoxide $\left(D_{\mathrm{LCO}}\right)$ were significantly lower in patients with DPLD than in patients with malignant lung tumours.

There were three patients with emphysematous lungs, though without pneumothoraces. One patient was diagnosed with an organising pneumonia and resultant respiratory failure, necessitating treatment with $1 \mathrm{~L} \cdot \mathrm{min}^{-1}$ of oxygen therapy via nasal cannula, which was started before the TBLC procedure. No other patients had respiratory failure before the TBLC. There were no patients with bleeding diathesis, chronic heart failure, severe renal dysfunction, or immunocompromise.

\section{Bronchoscopic intervention}

Procedure time, cryoprobe size, number and bronchus for TBLC, and sedation type are summarised in table 2 . The majority of patients underwent TBLC with a $1.9-\mathrm{mm}$ cryoprobe, with a freezing time of $6-7 \mathrm{~s}$ for the $1.9-\mathrm{mm}$ cryoprobe and $5 \mathrm{~s}$ for the $2.4-\mathrm{mm}$ cryoprobe. An average of 2.47 TBLCs per patient were performed. Lower lobes were more frequently chosen for TBLC than other lobes.

\section{Primary end-point}

Rate of severe or serious adverse events

Neither severe nor serious adverse events were identified, and there were no deaths. Therefore, the primary end-point was met in the present study.

\section{Secondary end-points}

\section{Rate of all adverse events}

The rate of mild and moderate adverse events related to bronchial bleeding, pneumothorax, pneumonia, and/or respiratory failure are summarised in table 3. There were no identified acute exacerbations of interstitial pneumonia. Nineteen patients (17\%) had no bronchial bleeding. Mild bronchial bleeding was identified in $67 \%$ of patients, and moderate bronchial bleeding was identified in $16 \%$ of patients. In patients who had moderate bronchial bleeding, there was total 41 cryobiopsies, and the median number of times of epinephrine-saline mixture administration was 3 times per cryobiopsy and the maximum number of times of epinephrine-saline mixture administration was 11 times per cryobiopsy. Mild pneumothoraces were identified in four patients (3.6\%), with spontaneous improvement. Only one patient (0.9\%) had a moderate pneumothorax necessitating chest drainage, and pneumonia and respiratory failure were observed in one patient. Three patients with emphysematous lungs did not have pneumothoraces after the

\section{TABLE 2 Bronchoscopic intervention}

Total ( $n=112)$

$\begin{array}{ll}\text { Procedure time min } & 34(18-71) \\ \text { TBLC } & \\ \text { Probe size } & 111(99) \\ 1.9 \mathrm{~mm} & 1(1) \\ 2.4 \mathrm{~mm} & 2.47 \\ \text { Cryobiopsies per patient } \mathrm{n} & \\ \text { Location of TBLC } & 33(11.9) \\ \text { Right upper lobe } & 19(6.9) \\ \text { Right middle lobe } & 131(47.3) \\ \text { Right lower lobe } & 21(7.6) \\ \text { Left upper lobe } & 73(26.4) \\ \text { Left lower lobe } & \\ \text { Sedation } & 35(17.5-35) \\ \text { Pethidine mg } & 8(2-20) \\ \text { Midazolam mg } & 0.09(0.04-0.9) \\ \text { Fentanyl mg } & \end{array}$

Data are presented as $\mathrm{n}(\%)$ or median (range), unless otherwise stated. DPLD: diffuse parenchymal lung disease; TBLC: transbronchial lung cryobiopsy. 
TABLE 3 Adverse events

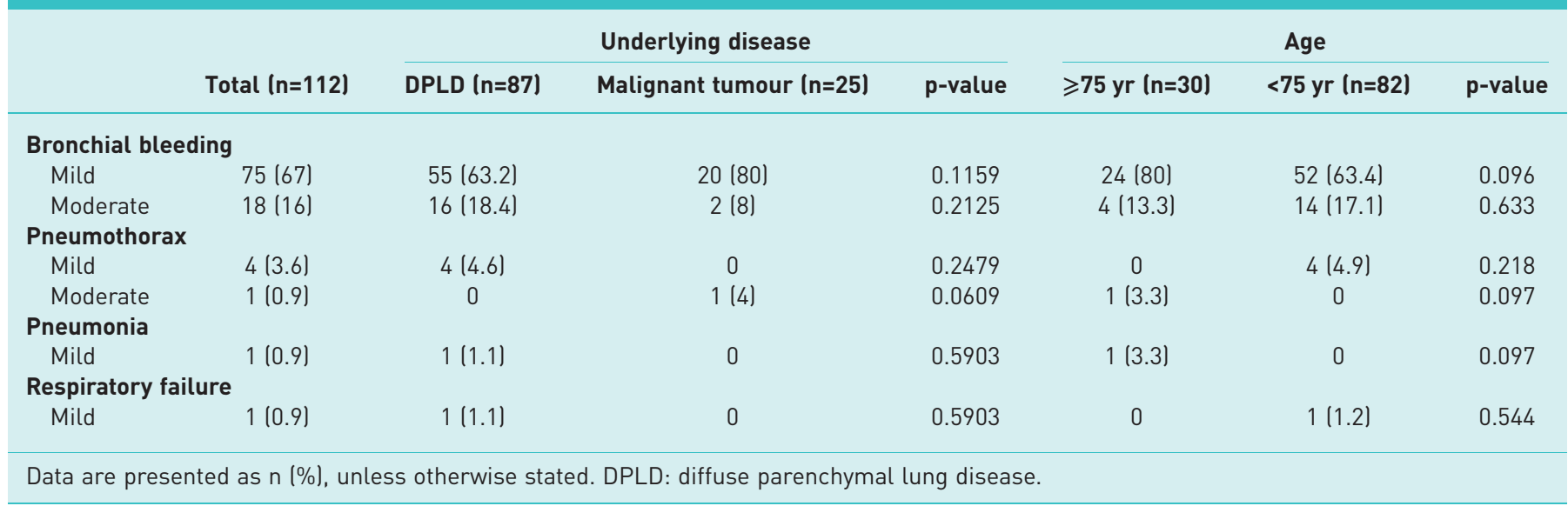

TBLC, and there were no patients who developed late pneumothoraces. There were no significant differences in the rates of adverse events between patients with DPLD and with malignant tumours.

Adverse events in elderly patients

The rate of adverse events in elderly patients aged $\geqslant 75$ years are shown in table 3 . The safety profile in these elderly patients was not significantly different from the safety profile in younger patients.

\section{Endobronchial balloon for prevention of bleeding}

Failure rates of selective bronchial blockade are summarised in table 4. After the cryoprobe and attached specimen were withdrawn and the bronchoscope was reinserted to evaluate for bronchial bleeding, the inflated endobronchial balloon was found to be displaced to different bronchi in 27 patients (9.7\%). Major displacements were observed in six patients $(2.2 \%)$, with displacements in both the upper and lower lobe bronchi observed in three cryobiopsies (1.1\%). Minor displacements were observed in 21 patients $(7.6 \%)$, with displacements in the upper, middle, and lower lobe bronchi in $1.8 \%, 1.1 \%$, and $4.7 \%$ of cryobiopsies, respectively. There were no significant differences in the frequencies of major or minor displacements between upper, middle, and lower lobes. The endobronchial balloon was ruptured in four cryobiopsies (1.4\%).

\section{Pathological evaluation and diagnosis}

The percentage of grades A and B specimens, which were determined to have value for evaluation, was 91.1\% in all patients (table 5). Grade B specimens were more common in patients with DPLD than in patients with malignant lung tumours. Definite and probable pathological diagnoses (levels A and B) were made in $84.9 \%$ of all patients, $86.2 \%$ of patients with DPLD, and $80 \%$ of patients with malignant lung tumours. Level A diagnoses were more commonly made in patients with malignant lung tumours, and

\section{TABLE 4 Endobronchial balloon for prevention of bleeding}

Total (n=277)

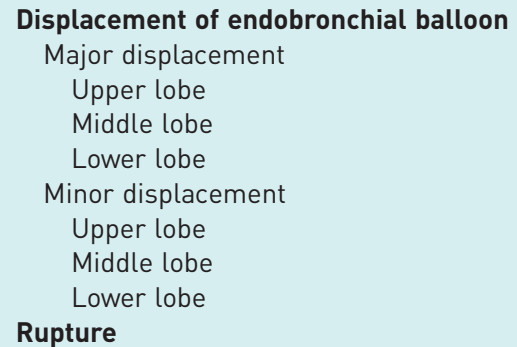




\begin{tabular}{|c|c|c|c|c|}
\hline & Total $(n=112)$ & DPLD (n=87) & Malignant tumour ( $n=25)$ & p-value \\
\hline \multicolumn{5}{|c|}{ Pathological quality and quantity } \\
\hline \multicolumn{5}{|c|}{ Quality score } \\
\hline Grade A & 63 (56.3) & 45 (51.7) & $18(72)$ & 0.717 \\
\hline Grade B & 39 (34.8) & $37(42.5)$ & $2(8)$ & 0.001 \\
\hline Grade C & $10(8.9)$ & $5(5.8)$ & $5(20)$ & 0.028 \\
\hline \multicolumn{5}{|c|}{ Pathological confidence } \\
\hline Level A & $46(41.1)$ & $28(32.2)$ & $18(72)$ & 0.001 \\
\hline Level B & 49 (43.8) & $47(54)$ & 2 (8) & $<0.001$ \\
\hline Level C & $17(15.1)$ & $12(13.8)$ & $5(20)$ & 0.446 \\
\hline Artefact & 3 (2.7) & $3(100)$ & 0 & 0.717 \\
\hline
\end{tabular}

level B diagnoses were more commonly made in patients with DPLD. There were pathological artefacts, including slight haemorrhages and intra-alveolar exudates, identified in only three cryobiopsies.

Multidisciplinary discussion diagnosis

MDD diagnoses are shown in table 6. The diagnostic yields of TBLCs for MDD diagnoses was 92\% in all patients, $95.4 \%$ in patients with DPLD, and $80 \%$ in patients with malignant lung tumours.

\section{Discussion}

This study is the first prospective investigation of the safety and utility of TBLCs in patients with diffuse or localised respiratory diseases in Japan. Definite or probable pathological diagnoses were made in $84.9 \%$ of obtained cryobiopsies, and MDD diagnoses were achieved in $92 \%$ of all patients. Mild to moderate bronchial bleeding was the most frequently observed complication of TBLC, and there were no severe or serious adverse events. The safety profile in elderly patients was not significantly different from that found in younger patients.

\section{TABLE 6 Multidisciplinary discussion diagnosis}

No. of patients

$\begin{array}{lc}\text { Diffuse parenchymal lung disease } & \\ \text { Idiopathic pulmonary fibrosis } & 13(11.6) \\ \text { Nonspecific interstitial pneumonia } & 6(5.4) \\ \text { Cryptogenic organising pneumonia } & 6(5.4) \\ \text { Acute fibrinous organising pneumonia } & 1(0.9) \\ \text { Unclassified interstitial pneumonia } & 13(11.6) \\ \text { Chronic hypersensitivity pneumonitis } & 9(8) \\ \text { Connective tissue disease associated interstitial lung disease } & 16(14.3) \\ \text { Smoking-related interstitial lung disease } & 1(0.9) \\ \text { Chronic eosinophilic pneumonia } & 4(3.6) \\ \text { Sarcoidosis } & 2(1.8) \\ \text { Pulmonary amyloidosis } & 1(0.9) \\ \text { Eosinophilic granulomatosis with polyangiitis } & 1(0.9) \\ \text { Drug-induced interstitial lung disease } & 4(3.6) \\ \text { Radiation pneumonitis } & 1(0.9) \\ \text { Diffuse alveolar haemorrhage } & 1(0.9) \\ \text { Multicentric Castleman disease } & 2(1.8) \\ \text { Viral pneumonia } & 1(0.9) \\ \text { Malignant tumour } & 17(15.2) \\ \text { Lung cancer } & 3(2.7)\end{array}$

Data are presented as $\mathrm{n}(\%)$. 
TBLC is a promising and safer alternative to surgical lung biopsies (SLB) in the diagnostic approach to DPLD [16, 17], and it appears sufficient for establishing the histological diagnosis of usual interstitial pneumonia [18]. TBLC also has a robust capability for identifying peripheral lung tumours [7]. The TBLC technique and protocols have not yet been standardised, however, and the reported complications associated with the procedure vary widely. Therefore, validation of the safety and utility of the TBLC procedure using an endobronchial balloon as a bleeding prophylaxis is needed.

Bleeding is the most common complication of TBLC. Serious bleeding during TBLC has been reported in up to $42 \%$ of procedures [9] and, in a previous meta-analysis, moderate bleeding was observed in $16.9 \%$ [19]. There is currently no standard severity scale for bronchial bleeding during TBLC. In the present study, the severity of bleeding was graded into four levels, with clinically insignificant mild or moderate bleeding the most frequently observed severity levels. While severe and serious bleeding during TBLC can lead to life-threatening complications and extended hospital stays, there were no cases of severe or serious bleeding identified in our study. We also did not identify any other severe or serious adverse events or deaths in the study population, demonstrating the acceptable safety profile of the TBLC protocol for diagnosing DPLD and malignant tumours in the lung periphery.

The endobronchial ballooning procedure using the Fogarty balloon was shown to be efficacious for the prevention of bleeding in the present study; however, haemostasis sometimes failed due to displacement or rupture of the endobronchial balloon. Although there is a known risk of displacement of the endobronchial balloon in the upper lobe [20], we also identified displacements in the middle and lower lobes. There were, however, no significant differences identified in the frequency of displacements in different lobes in the present study. This study is the first to report a displacement rate for the endobronchial balloon. Our study shows that displacements may occur just after the withdrawal of the cryoprobe and the bronchoscope because the cryoprobe with the attached lung specimen can easily contact the endobronchial balloon, leading to displacement.

Guidance of the endobronchial balloon to the upper lobe bronchi was sometimes difficult, and there was a risk of displacement to the upper lobe. Accordingly, the lower lobe bronchi were more frequently chosen for TBLC, and this choice may have affected the frequency of bleeding complications.

Rupture of the endobronchial balloon has been demonstrated in one previous case report and is an uncommon, though important, complication of TBLC [21]. In the present study, balloon rupture was observed in four patients (1.4\%), and this study is the first to report the frequency of balloon rupture during TBLC. In the present study, the amount of air injected into the endobronchial balloon was fixed; therefore, excessive pressure was not a likely cause for the observed ruptures. The observed ruptures of the endobronchial balloon may have been due to accidental contact between the cryoprobe and the endobronchial balloon.

Although major displacements and ruptures of the endobronchial balloon were observed, there were no severe or serious bleeding events, potentially due to the haemostatic effect of the prophylactic and routine use of the endobronchial balloon. Therefore, careful use and accurate positioning of the endobronchial balloon are considered important steps to minimise the risk of bronchial bleeding during the TBLC procedure.

There may also have been procedural factors that influenced our end-point of bronchial bleeding. In some patients, radial endobronchial ultrasound signals were used to identify blood vessels, which helped us to choose biopsy sites and may, therefore, have affected the frequency of bleeding complications. In addition, there was only one patient who underwent TBLC with a $2.4-\mathrm{mm}$ cryoprobe. Since the incidence of bronchial bleeding in patients undergoing TBLCs may be higher with use of a 2.4-mm cryoprobe than with a $1.9-\mathrm{mm}$ cryoprobe, our choice of cryoprobe size might also have affected the frequency of bleeding complications.

Pneumothorax has been reported as a major complication of TBLC, with an incidence rate of up to $26 \%$ $[9,17]$. A previous meta-analysis reported an average incidence rate of pneumothorax during the TBLC procedure of $20.2 \%$, with $15.5 \%$ requiring drainage [19]. In our study, the incidence rate of pneumothorax was $4.4 \%$, with only one patient $(0.9 \%)$ requiring chest drainage. All the pneumothoraces in our study were mild or moderate in severity and were improved without clinical deterioration. An increased prevalence of pneumothoraces has been reported with freezing times of 5 or $6 \mathrm{~s}$ [22]; however, the present protocol demonstrated safety with freezing times of 5 to $7 \mathrm{~s}$.

Previous studies reported performance of the TBLC procedure in patients over 75 years of age [8, 23], with no suggested age limit [9]. Patients $\geqslant 75$ years of age were included in the present study, and their safety profile was not significantly different from other patients, suggesting that this protocol may be acceptable even for elderly patients. 
There were significant differences between the baseline pulmonary function tests between patients with DPLD and malignant tumours; however, the safety profile was not significantly different between these two groups. Therefore, our TBLC protocol can be considered safe in patients with both diffuse and localised respiratory diseases.

The diagnostic yield of TBLCs for DPLDs has been shown to vary from 50.6 to $100 \%$ in previous studies $[2,9,24]$. In addition, the diagnostic accuracy of TBLC has been previously assessed by evaluation of the agreement between SLB based on histopathological analyses and multidisciplinary discussions of diagnosis [16]. Histopathological interpretations and MDD diagnoses using TBLCs or SLBs have previously been shown to have high levels of agreement [25]. In the present study, the diagnostic yield of TBLCs for MDD diagnoses of DPLDs was high; however, the present study did not evaluate SLB specimens. Instead, pathological quality and confidence were categorised into three groups according to previous reports [8, 15], and a definite or probable pathological diagnosis (levels $\mathrm{A}$ or B) was made in $86.2 \%$ of DPLD patients.

In patients with malignant lung tumours, the utility of TBLC for diagnosis can depend on whether TBLC specimens include malignant cells. The diagnostic yield of TBLC for peripheral lung cancer has been reported to be $68 \%$ [26] or $81.1 \%$ [27] in previous studies. In our study, malignant tumours in the lung periphery were diagnosed pathologically in $80 \%$ of cryobiopsies, and these rates were consistent with previous reports $[7,27]$. In addition to an adequate safety profile, we also showed that the diagnostic yield of TBLC is acceptable in patients with both diffuse and localised respiratory diseases.

The present study had several limitations. First, there was no control group who underwent TBLC without the use of an endobronchial balloon. Further studies that compare the safety of the procedure with and without use of an endobronchial balloon are needed. Second, we did not confirm diagnoses through comparison with SLBs; therefore, we could not compare pathological diagnoses made by this procedure for each patient. Finally, there may be a potential selection bias because a pulmonologist determined the necessity for the procedure and enrolment into the study.

\section{Conclusion}

This prospective multicentre study demonstrated that TBLC can safely be utilised for diagnoses of diffuse and localised respiratory diseases, even in elderly patients. Furthermore, careful endobronchial balloon placement and usage were demonstrated to be important steps in minimising the risk of bronchial bleeding during TBLC. Using our protocol, the safety profile and diagnostic yield of TBLC were shown to be acceptable.

Conflict of interest: None declared.

\section{References}

1 Babiak A, Hetzel J, Krishna G, et al. Transbronchial cryobiopsy: a new tool for lung biopsies. Respiration 2009; 78: 203-208.

2 Pajares V, Puzo C, Castillo D, et al. Diagnostic yield of transbronchial cryobiopsy in interstitial lung disease: a randomized trial. Respirology 2014; 19: 900-906.

3 Hetzel J, Hetzel M, Hasel C, et al. Old meets modern: the use of traditional cryoprobes in the age of molecular biology. Respiration 2008; 76: 193-197.

4 Ravaglia C, Wells AU, Tomassetti S, et al. Transbronchial lung cryobiopsy in diffuse parenchymal lung disease: comparison between biopsy from 1 segment and biopsy from 2 segments - diagnostic yield and complications. Respiration 2017; 93: 285-292.

5 Fruchter O, Fridel L, El Raouf BA, et al. Histological diagnosis of interstitial lung diseases by cryo-transbronchial biopsy. Respirology 2014; 19: 683-688.

6 Tomassetti S, Wells AU, Costabel U, et al. Bronchoscopic lung cryobiopsy increases diagnostic confidence in the multidisciplinary diagnosis of idiopathic pulmonary fibrosis. Am J Respir Crit Care Med 2016; 193: 745-752.

7 Sanchez-Cabral O, Martinez-Mendoza D, Fernandez-Bussy S, et al. Utility of transbronchial lung cryobiopsy in non-interstitial diseases. Respiration 2017; 94: 285-292.

8 Kuse N, Inomata M, Awano N, et al. Management and utility of transbronchial lung cryobiopsy in Japan. Respir Investig 2019; 57: 245-251.

9 Hetzel J, Maldonado F, Ravaglia C, et al. transbronchial cryobiopsies for the diagnosis of diffuse parenchymal lung diseases: expert statement from the Cryobiopsy Working Group on Safety and Utility and a call for standardization of the procedure. Respiration 2018; 95: 188-200.

10 Hetzel J, Eberhardt R, Petermann C, et al. Bleeding risk of transbronchial cryobiopsy compared to transbronchial forceps biopsy in interstitial lung disease - a prospective, randomized, multicentre cross-over trial. Respir Res 2019; 20: 140 .

11 Echevarria-Uraga JJ, Pérez-Izquierdo J, García-Garai N, et al. Usefulness of an angioplasty balloon as selective bronchial blockade device after transbronchial cryobiopsy. Respirology 2016; 21: 1094-1099.

12 Baron TH, Kamath PS, McBane RD. Management of antithrombotic therapy in patients undergoing invasive procedures. N Engl J Med 2013; 368: 2113-2124. 
13 Takemura T, Baba T, Niwa T, et al. Histology for Transbronchial Lung Cryobiopsy Samples. In: Poletti V, ed. Transbronchial cryobiopsy in diffuse parenchymal lung disease. Cham, Springer, 2019.

14 Dindo D, Demartines N, Clavien P-A. Classification of surgical complications: a new proposal with evaluation in a cohort of 6336 patients and results of a survey. Ann Surg 2004; 240: 205-213.

15 Takashi N, Tamiko T, Erina T, et al. Initial report of transbronchial lung cryobiopsy for diffuse parenchymal lung disease. J Japan Soc Respir Endosc 2018; 40: 453-458.

16 Troy LK, Grainge C, Corte T, et al. Cryobiopsy versus open lung biopsy in the diagnosis of interstitial lung disease (COLDICE): protocol of a multicentre study. BMJ Open Respir Res 2019; 6: e000443.

17 Iftikhar IH, Alghothani L, Sardi A, et al. Transbronchial lung cryobiopsy and video-assisted thoracoscopic lung biopsy in the diagnosis of diffuse parenchymal lung disease. a meta-analysis of diagnostic test accuracy. Ann Am Thorac Soc 2017; 14: 1197-1211.

18 Lentz RJ, Taylor TM, Kropski JA, et al. Utility of flexible bronchoscopic cryobiopsy for diagnosis of diffuse parenchymal lung diseases. J Bronchology Interv Pulmonol 2018; 25: 88-96.

19 Ravaglia C, Bonifazi M, Wells AU, et al. Safety and diagnostic yield of transbronchial lung cryobiopsy in diffuse parenchymal lung diseases: a comparative study versus video-assisted thoracoscopic lung biopsy and a systematic review of the literature. Respiration 2016; 91: 215-227.

20 Kodati R, Sehgal IS, Prasad KT, et al. Acute exacerbation and progression of interstitial lung disease after transbronchial lung cryobiopsy. J Bronchology Interv Pulmonol 2019; 26: e68-e72.

21 Mittal S, Hadda V, Mohan A, et al. Rupture of occlusion balloon during transbronchial lung cryobiopsy. Lung India 2019; 36: 263-265.

22 Ing $\mathrm{M}$, Oliver RA, Oliver BG, et al. Evaluation of transbronchial lung cryobiopsy size and freezing time: a prognostic animal study. Respiration 2016; 92: 34-39.

23 Cooley J, Balestra R, Aragaki-Nakahodo AA, et al. Safety of performing transbronchial lung cryobiopsy on hospitalized patients with interstitial lung disease. Respir Med 2018; 140: 71-76.

24 Fruchter O, Fridel L, Rosengarten D, et al. Transbronchial cryo-biopsy in lung transplantation patients: first report. Respirology 2013; 18: 669-673.

25 Troy LK, Grainge C, Corte TJ, et al. Diagnostic accuracy of transbronchial lung cryobiopsy for interstitial lung disease diagnosis (COLDICE): a prospective, comparative study. Lancet Respir Med 2020; 8: 171-181.

26 Schuhmann M, Bostanci K, Bugalho A, et al. Endobronchial ultrasound-guided cryobiopsies in peripheral pulmonary lesions: a feasibility study. Eur Respir J 2014; 43: 233-239.

27 Nasu S, Okamoto N, Suzuki H, et al. Comparison of the utilities of cryobiopsy and forceps biopsy for peripheral lung cancer. Anticancer Res 2019; 39: 5683-5688. 